\title{
Coating and Characterization of Mock and Explosive Materials
}

\author{
Emily M. Hunt and Matt Jackson \\ Department of Engineering and Computer Science, West Texas A\&M University, Canyon, TX 79016, USA \\ Correspondence should be addressed to Emily M. Hunt, ehunt@wtamu.edu
}

Received 16 February 2012; Revised 31 May 2012; Accepted 1 June 2012

Academic Editor: J. Paulo Davim

Copyright ( 2012 E. M. Hunt and M. Jackson. This is an open access article distributed under the Creative Commons Attribution License, which permits unrestricted use, distribution, and reproduction in any medium, provided the original work is properly cited.

\begin{abstract}
This project develops a method of manufacturing plastic-bonded explosives by using use precision control of agglomeration and coating of energetic powders. The energetic material coating process entails suspending either wet or dry energetic powders in a stream of inert gas and contacting the energetic powder with atomized droplets of a lacquer composed of binder and organic solvent. By using a high-velocity air stream to pneumatically convey the energetic powders and droplets of lacquer, the energetic powders are efficiently wetted while agglomerate drying begins almost immediately. The result is an energetic powder uniformly coated with binder, that is, a PBX, with a high bulk density suitable for pressing. Experiments have been conducted using mock explosive materials to examine coating effectiveness and density. Energetic materials are now being coated and will be tested both mechanically and thermally. This allows for a comprehensive comparison of the morphology and reactivity of the newly coated materials to previously manufactured materials.
\end{abstract}

\section{Introduction}

The study of explosives is of considerable importance because of their many technological and defense-related applications. Plastic-bonded explosives (PBXs) are a mixture of particulate energetic material and a polymer binder. PBXs have been commonly used in both military and industry because of their improved safety, enhanced mechanical properties, and reduced vulnerability during storage and transportation [1].

Mechanical initiation of PBXs is strongly dependent on the heterogeneity of the energetic materials and polymeric binder at the mesoscale $[2,3]$. The polymer binder in the PBXs plays an important role in enhancing the properties of the base explosive (i.e., improving mechanical properties, enhancing chemical and thermal stability, reducing environmental impact, etc.). Therefore, there is an increasing need to understand and predict the chemical, thermal, and mechanical properties of the PBXs as well as the interactions among the energetic particles and polymeric binder [4]. Although many studies have been conducted on composite explosives to examine the effects of such parameters as particle size [5], crystal defects [6], and binder properties [7], the contributions of grain anisotropy, defects and interfacial responses to hot-spot initiation still remain very difficult task.

This study examines a method to develop PBX in which explosive powder is bound together in a matrix using small quantities (typically $5-10 \%$ by weight) of a synthetic polymer or plastic. PBXs are normally used for explosive materials that are otherwise difficult to form. This new method is called precision coating of high-explosive (HE) powders because we are using a more specific and precise method to incorporate the plastic into the explosive matrix. This new method will provide a safer, more economical method to produce an even higher-quality PBX. Explosive processing safety is enhanced by the fact that only a small quantity of explosives is being very rapidly coated at any one time within the system. The focus of this study will be on testing and characterization before and after coating using SEM and touch probe technology which is just an optical 3D measurement device which will allow us to actually "see" a $3 \mathrm{D}$ view of each coated particle. The newly coated and characterized powders will be tested for mechanical properties such as density of coating with relation to thickness and density of pressing and behavior. This will allow a 


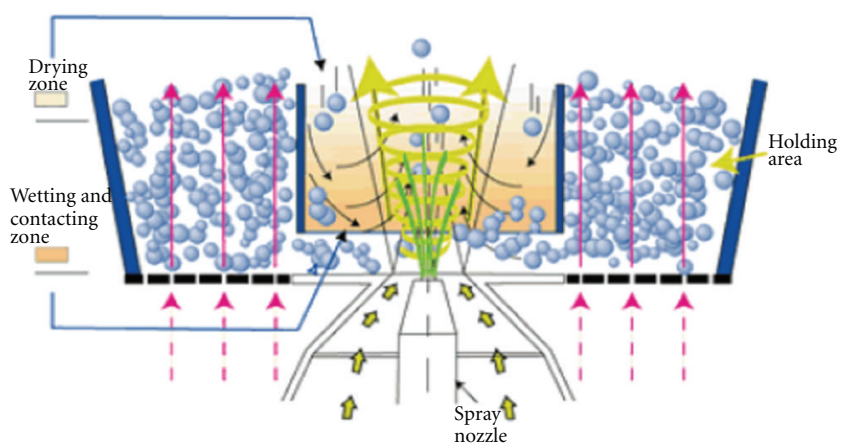

High-velocity air

- Granulation liquid

_ Low-velocity air

FIGURE 1: Schematic of precision coating process.

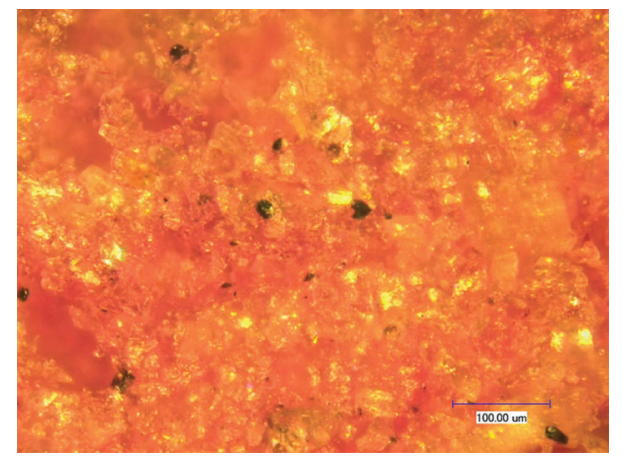

Figure 2: LM-17 mock HE processed in the Strea-1 precision coater.

comprehensive comparison of the morphology and reactivity of the newly coated mock HE to previously manufactured materials.

\section{Experimental}

The HE coating process entails suspending either wet or dry explosive powders in a stream of inert gas and contacting the explosive powder with atomized droplets of a lacquer composed of binder and organic solvent. By using a highvelocity air stream to pneumatically convey the explosive powders and droplets of lacquer, the explosive powders will be efficiently wetted while agglomerate drying will begin almost immediately. The intended result is an explosive powder uniformly coated with binder, that is, a PBX, with a high bulk density suitable for pressing. In other words, we will have explosive particles that are coated in a homogeneous thickness of plastic rather than the plastic just being mixed or "stirred" into the explosive powders. The precision agglomeration process is shown in Figure 1.

A Strea-1 precision coating unit was purchased from Aeromatic-Fielder for using in the precision coating experiments. Two mock HEs were coated: LM-17 and K-F800 and are shown in Figure 2. The dark spots represent individual crystals that were stained to give an idea of the relative

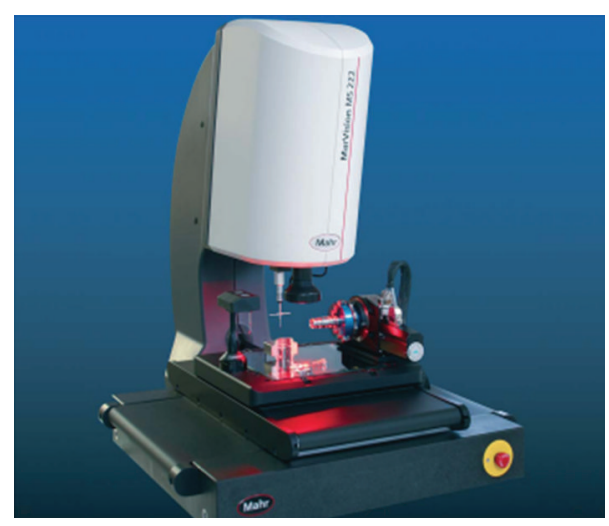

Figure 3: MarVision MS 222 (informational booklet).

particle size, and the light spots are just brightness caused by reflective light of the microscope.

A method for testing the completeness of the coating on particles which is characterized by how much of the actual particle is coated in plastic is also developed for a better understanding of pellet pressing capabilities and the bulk density of the coated HE. Once the mock HE has been coated or completely covered in the plastic and the coating has been analyzed using the metrology equipment described above as well as microscopy, the actual characterization of the mechanical and thermal properties of the newly coated mock HE or the mock explosive crystals that have been covered in plastic using the precision coating process is conducted as described below. Most initial tests were conducted with standard salt samples. These were the simplest and cheapest to work with and allowed equipment parameters to be evaluated and the test procedure to be optimized. Some compositions seem much better suited to this method, as will be seen in the results below.

Bulk density of the coated samples is examined using the standard density tests developed at B\&W Pantex Plant in Amarillo, TX, USA. The sample size is $200 \pm 0.1$ grams weighed to the nearest 0.01 gram. The material is placed into a previously tared $250 \mathrm{~mL}$ graduated cylinder. The material is compacted and then the graduated cylinder is placed into a wooden box with the following inside dimensions: $102 \times$ $102 \times 102 \mathrm{~mm}(4 \times 4 \times 4$ inch $)$. The inside top and bottom of the box are padded with $6.35 \mathrm{~mm}(0.25 \mathrm{inch})$ rubber foam. A hole is cut in the hinged top to fit a $250 \mathrm{~mL}$ graduated cylinder. The cylinder is raised to the top of the box and then released. This is repeated 20 times at the rate of 1 drop per second. The bulk volume is read and recorded.

This study tests and validates the use of metrology equipment to characterize the uniformity of coating as a quality assurance means of maximizing high bulk density suitable for pressing. The MarVision MS 222 (Figure 3) is an optical 3D measuring technology which will allow us to actually "see" a 3D view of each coated particle by mapping the surface of the particle with a laser.

The impact sensitivity of the coated HE is investigated using a modified type 12 impact tester (KeyKinetics, Inc.). Tests are also conducted using both powdered and pressed 


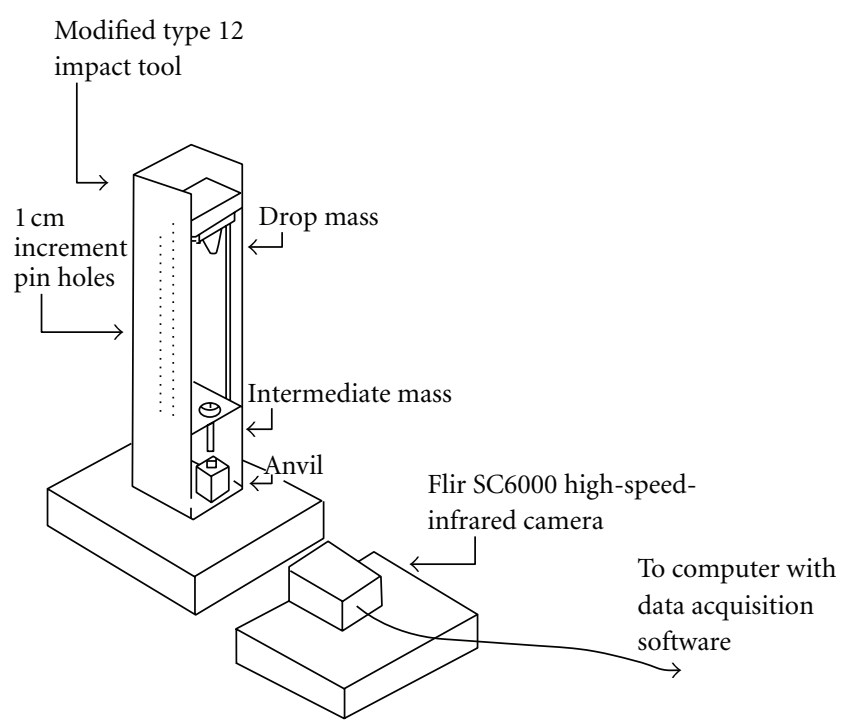

FIgUre 4: Modified type 12 impact tester [8].

samples of the coated HE. The impact tester (Figure 4) has a drop mass of $1 \mathrm{~kg}$ with an intermediate mass of $1.5 \mathrm{~kg}$. Each test is conducted with a $30 \pm 5 \mathrm{mg}$ powder sample or pressed sample placed on the anvil. The intermediate mass is rested on the specimen, and the drop mass makes contact with the top of the intermediate mass (Figure 4).

The drop mass is released from a variable height, and the infrared camera is used to determine ignition based on a go/no-go determination. Once an optimal range is found in which there are positive and negative reactions, tests are conducted on $1 \mathrm{~cm}$ intervals. Using an abbreviated Bruceton method which is typically accepted as the standard in explosives experimentation, the lowest drop height that produces a greater than $50 \%$ reaction is used to calculate ignition energy using a standard equation for potential energy (1) as follows:

$$
\mathrm{PE}=m g h,
$$

where PE is the potential energy, $m$ is the mass of the drop mass, and $h$ is the optimal height for reaction. The infrared camera is used to determine the velocity of the drop mass as it travels to the sample. The velocity is then used to calculate the kinetic energy of the sample (2). The high-speed capabilities of the high-speed infrared camera or SC6000 (4000 fps) is also an effective tool to measure the time between initial contact and ignition of the sample:

$$
\mathrm{KE}=\frac{1}{2} m v^{2},
$$

where KE is the kinetic energy, and $v$ is the velocity of the drop mass. This test provides a means by which the visible signs of reaction such as emission of heat by the infrared camera (Figure 5) can be related to impact properties, such as impact speed or impact stress. Figure 5 shows a positive reaction occurring during an impact test. Visible signs of reaction are occurring between the anvil and drop weight as the heat and light are "shooting" out of the impact.

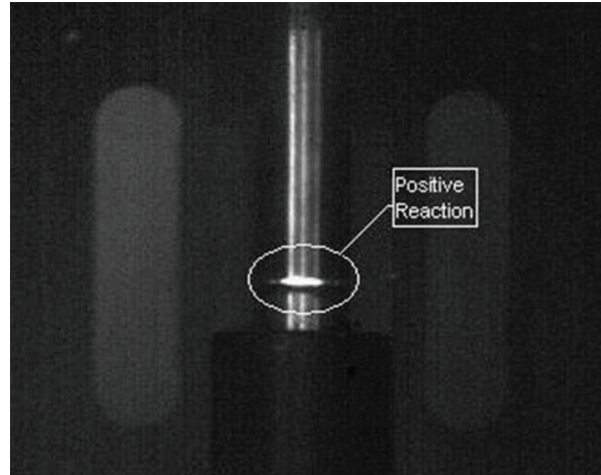

Figure 5: Visible signs of reaction [9].

TABLE 1: Bulk density testing results.

\begin{tabular}{cccc}
\hline & Initial $\left(\mathrm{g} / \mathrm{cm}^{3}\right)$ & Final $\left(\mathrm{g} / \mathrm{cm}^{3}\right)$ & Increase $(\%)$ \\
\hline LM-17 & & & \\
A & 0.419 & 0.474 & 13.2 \\
B & 0.520 & 0.700 & 34.6 \\
K-F800 & & & \\
A & 0.254 & 0.278 & 9.3 \\
B & 0.208 & 0.238 & 14.3 \\
\hline
\end{tabular}

\section{Results and Discussion}

The following Table 1 shows the results from the bulk density testing. Method A is the traditional method where the plastic is just mixed or "stirred" into the explosive powders and B is the precision coating method.

Initial bulk density tests yield results for the newly coated mock high-explosive material (B) that are similar to mock high-explosive material using previous methods (A). Since the methods for coating mock can produce different particle sizes due to inconsistencies in the application process, future density testing will include grinding the mock to produce a consistent powder and repeatable tests. In this way, the density testing would consist of particles that were similar in size, and any differences could be attributed to the mock/plasticizer distribution.

In general, this process seems best suited to the mock mixtures using the K-F800 binder. Bulk densities as high as $0.9 \mathrm{~g} / \mathrm{cm}^{3}$ were obtained in the mock HE using the method for density measurement described above but can be reasonably expected to be $0.6-0.7 \mathrm{~g} / \mathrm{cm}^{3}$ on average. With salt, bulk densities over $1 \mathrm{~g} / \mathrm{cm}^{3}$ are possible using the method described above, but $0.9-1 \mathrm{~g} / \mathrm{cm}^{3}$ is the average. Product recovery or the amount of useable coated mock HE powders that is recovered from the precision coating process varies greatly and continues to improve and currently the average amount of mock HE recovery is $70-80 \%$.

The biggest factor that affects the bulk density is the thickness of the binder/acetone mixture. When the mixture is too thick, the nitrogen pressure that forces the mixture through the nozzle has to be greatly increased. This causes the binder to be sprayed right to the bottom of the fluid 
LM-17
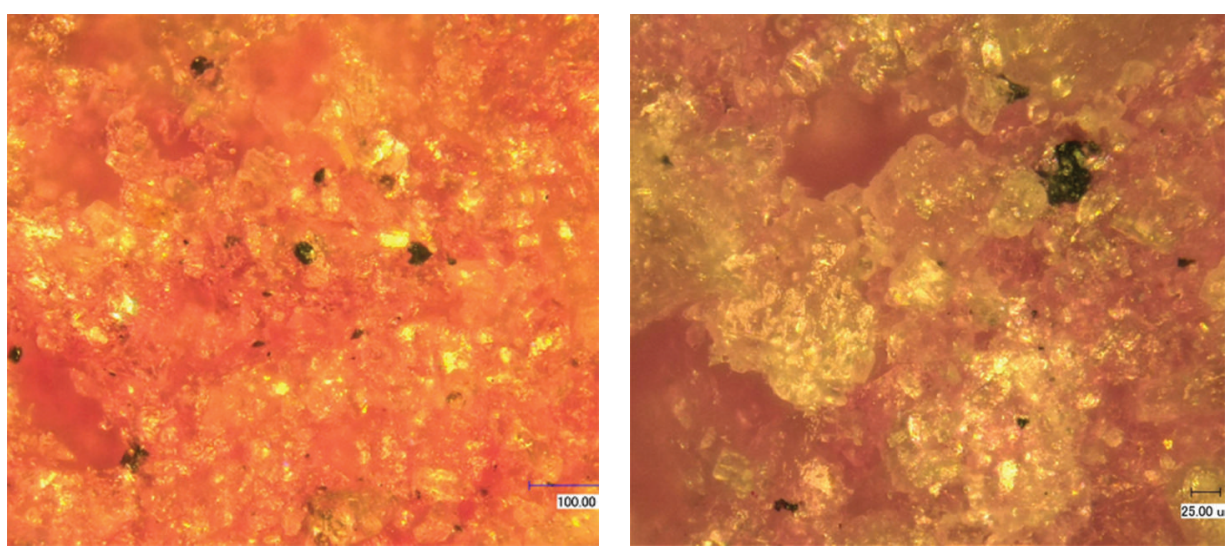

(a)

LM-17

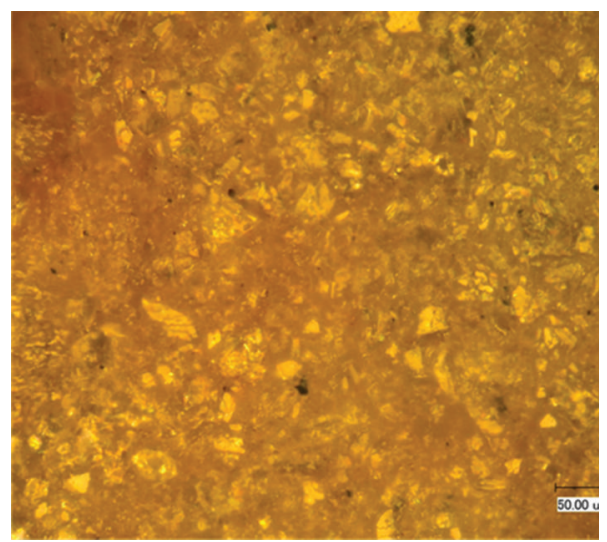

K-F800

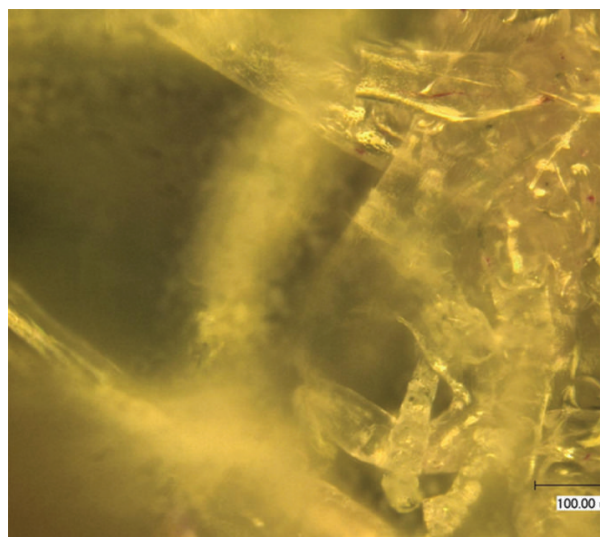

(b)

FIGURE 6: Mock PBX created by (a) amination and (b) precision coating.

bed heavily coating the materials at the bottom. When this occurs, large chunks develop that greatly reduce the bulk density. If the mixture is too thin, it will delay evaporation which weighs the material down and also causes clumping at the bottom. At this point in the experimentation process, it has been determined that if the mixture is fully dissolved (overnight works best), stirred slowly for a cycle of 10 to 12 minutes, and introduced 5 to 6 minutes in the cycle the least clumping occurs which yields the highest bulk densities.

Temperature and air flow through the system also have a significant effect because they are the two most easily controlled parameters in the precision coating process using the Strea-1 precision coater. High temperatures must be used for both the mixture and the mock product. The highest setting, which is $90^{\circ} \mathrm{C}$, has been used to the greatest effect. If the air flow is too high, the material is blown into the filters and nothing gets coated. If it is too low, the material just sits on the bottom and there is a sticky mess. The mock material works best with 45-50\% air flow through the system while the salt works best with $60-80 \%$ air flow. For best results, it is necessary to achieve a binder/acetone mixture at a high-temperature setting and a moderate air flow setting.
LM-17 and K-F800 have successfully been processed using the precision coating process described in the experimental section above according to microscopy and density results. Figure 6 shows pictures taken by an optical microscope of the coated mixtures. There are two coated mixtures for each type of mock HE, the first being processed through a traditional method (A) and the second processed in the precision coater $(\mathrm{B})$.

The LM-17 mixtures contain spherical mock HE (or salt) particles and appear to be homogenous. The K-F800 mixture produced by the traditional method appears to also produce spherical, homogenous samples, while the precision coated sample shows cylindrical crystal structures as well. Since the method of applying the plastic is different, it is expected that they will produce a different shape regardless of the starting material. The physical properties of the plastic itself are controlled in the precision coating process using temperature and airflow. The implications of these results should be examined during future mechanical testing of the coated samples.

Preliminary tests of PBX 9502 have been conducted and will be expanded upon in further study. All of the tests were on 10-gram samples except the last one, which was 10.5 
grams - increasing the binder percentage. The first four tests were run using the $95 / 5$ ratio of TATB to binder specified by B\&W Pantex. The fifth test used a 90.5/9.5 ratio and was run using the previously recovered samples. The TATB was reused due to limits on supply. The five tests had losses ranging from $44.4 \%$ to $100 \%$ which means that there was no product left to recover and producing seemingly uncoated TATB. Small changes were made including temperature, acetone, and binder variation attempting to produce better results with little success.

Recent research of fluidized bed coating methods revealed an approach using very large sample sizes, and these methods were modified for use in the coater. Due to the limit of TATB supply, talc or chalk-like powder was used in its place because of particle/powder similarity. The first test ran with talc was particularly successful. A sample size of 210 grams was used with a loss of only $7.51 \%$. In addition, the product was coated well. For the second test, the amount of binder and acetone was doubled to attempt to get even better coating. The resulting product was wet but coated extremely well with a loss of about $30 \%$. The third and last test using talc was run more similar to the first with a result of decent coating and loss of about $14 \%$. Thus far, it seems that larger sample sizes are going to yield better results based purely on the amount of useable, coated HE powder that was recovered from the precision coating process. Based on these results, it is recommended that tests in the near future consist of larger salt and mock HE samples which can be compared to past results. Bulk density results on these larger samples are also in the target range (specified by B\&W Pantex) of $0.4-0.6 \mathrm{~g} / \mathrm{cm}^{3}$ for mocks and salt.

Finally, and perhaps most importantly, recent results show much better results for all sample types with drastically increased sample sizes (up to 100 grams). This includes a decrease in loss from as high as $90 \%$ to as low as $4 \%$ while not sacrificing the integrity of the results.

\section{Conclusion}

The percentage increase in bulk density of LM-17 is improved a factor of 2.5 and that of K-F800 is improved by approximately 1.5 times than that of traditional methods as seen in the previous table using the density measurement methods described in the experimental section. An examination of the effect of particle size and coating thickness or time on the mechanical and thermal properties of the samples is needed for further explanation. The next part of this project will be to coat the actual HE using the precision coater. The experimental tests described in this paper will be used to characterize the PBX both mechanically and thermally. These results will be used to compare this new method for developing PBX to traditional methods.

\section{Acknowledgments}

The authors would like to acknowledge the support from B\&W Pantex Plant in Amarillo, TX, USA, as well as from research assistants Kyle Davis and Matt Dolezal.

\section{References}

[1] T. R. Gibbs and A. Popolato, LASL Explosive Property Data, University of California Press, Berkeley, Cailf, USA, 1980.

[2] J. G. Bennett, K. S. Haberman, J. N. Johnson, B. W. Asay, and B. F. Henson, "A constitutive model for the non-shock ignition and mechanical response of high explosives," Journal of the Mechanics and Physics of Solids, vol. 46, no. 12, pp. 2303-2322, 1998.

[3] J. K. Dienes, Q. H. Zuo, and J. D. Kershner, "Impact initiation of explosives and propellants via statistical crack mechanics," Journal of the Mechanics and Physics of Solids, vol. 54, no. 6, pp. 1237-1275, 2006.

[4] W. Zhu, J. Xiao, W. Zhu, and H. Xiao, "Molecular dynamics simulations of RDX and RDX-based plastic-bonded explosives," Journal of Hazardous Materials, vol. 164, no. 2-3, pp. 1082-1088, 2009.

[5] H. Tan, Y. Huang, C. Liu, G. Ravichandran, H. M. Inglis, and P. H. Geubelle, "The uniaxial tension of particulate composite materials with nonlinear interface debonding," International Journal of Solids and Structures, vol. 44, no. 6, pp. 1809-1822, 2007.

[6] P. J. Rae, "Studies of the failure mechanisms of polymer bonded explosives by high resolution moiré interferometry and environmental scanning electron microscopy," in Proceedings of the 11th Detonation Symposium, September 1998.

[7] C. M. Cady, W. R. Blumenthal, G. T. Gray, and D. J. Idar, "Mechanical properties of plastic-bonded explosive binder materials as a function of strain-rate and temperature," Polymer Engineering and Science, vol. 46, no. 6, pp. 812-819, 2006.

[8] E. M. Hunt and M. L. Pantoya, "Impact sensitivity of intermetallic nanocomposites: a study on compositional and bulk density," Intermetallics, vol. 18, no. 8, pp. 1612-1616, 2010.

[9] E. M. Hunt, S. Malcolm, M. L. Pantoya, and F. Davis, "Impact ignition of nano and micron composite energetic materials," International Journal of Impact Engineering, vol. 36, no. 6, pp. 842-849, 2009. 

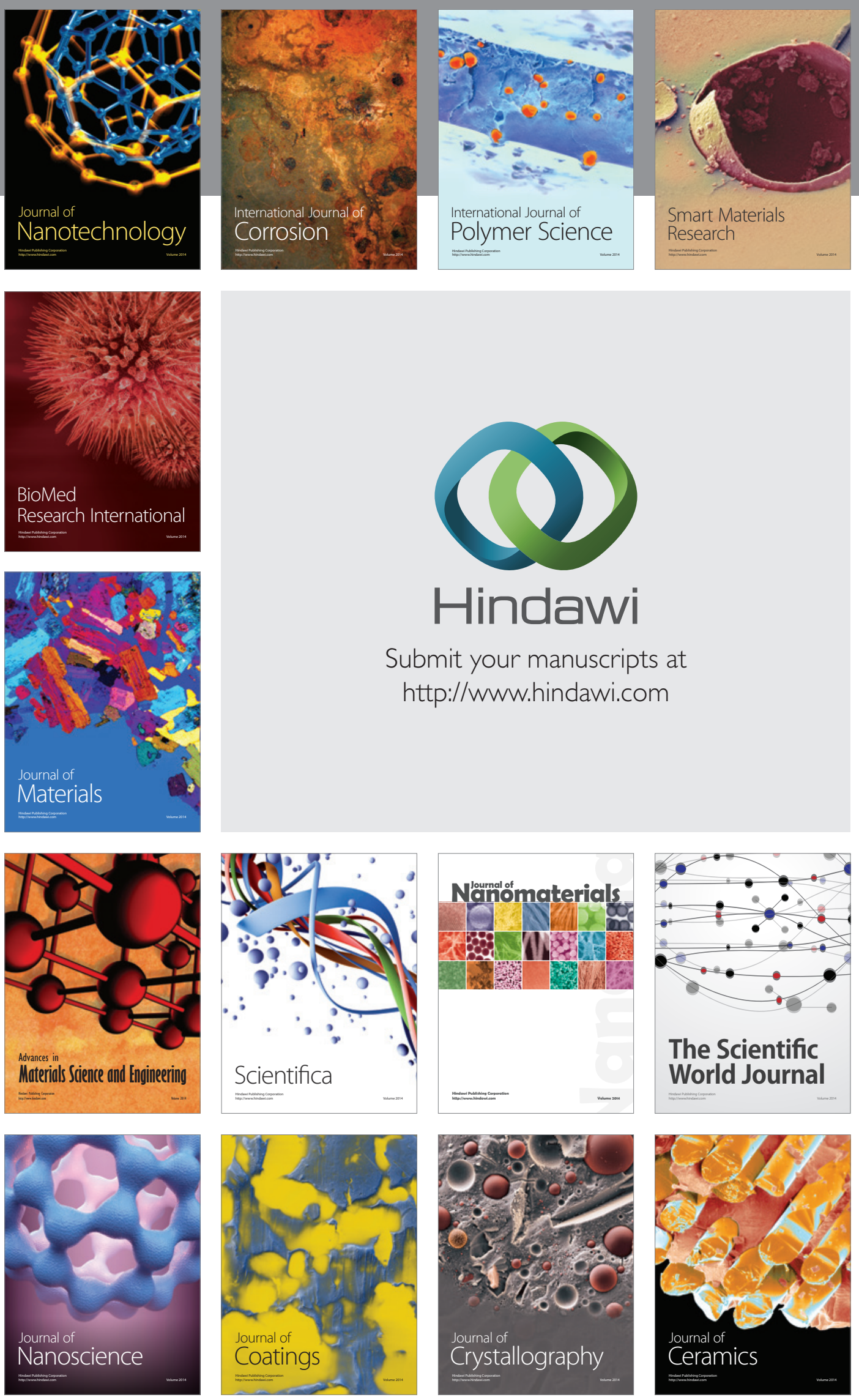

The Scientific World Journal

Submit your manuscripts at

http://www.hindawi.com

\section{World Journal}

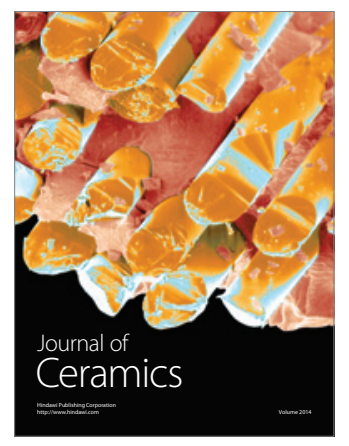

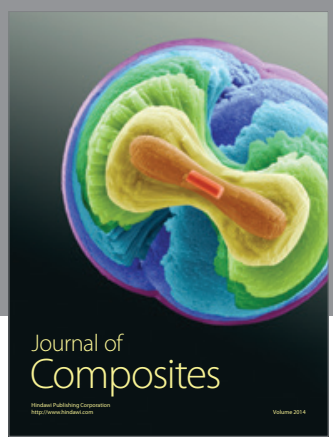
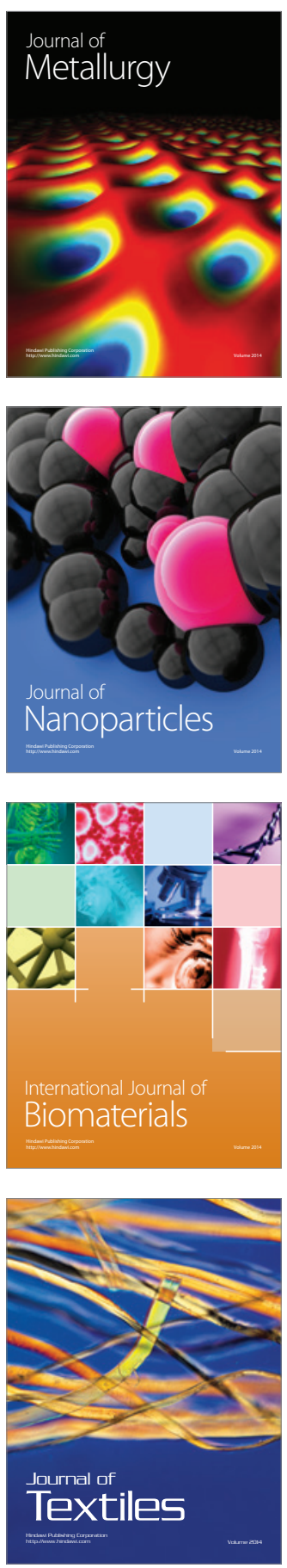